九州大学学術情報リポジトリ

Kyushu University Institutional Repository

\title{
LOCAL MAJORITY TRANSFORMATIONS IN CELL SPACE : INFORMATION SCIENCE APPROACH TO BIOMATHEMATICS, II
}

Kitagawa, Toshio

Research Institute of Fundamental Information Science, Faculty of Science, Kyushu University

Yamaguchi, Masako

Research Institute of Fundamental Information Science, Faculty of Science, Kyushu University

https://doi.org/10.5109/13053

出版情報: 統計数理研究. 14 (3/4)，pp.61-82，1971-03. Research Association of Statistical Sciences

バージョン：

権利関係 : 


\title{
LOCAL MAJORITY TRANSFORMATIONS IN CELL SPACE -INFORMATION CIENCE APPROACH TO BIOMATHEMATICS, II
}

\author{
By \\ Tosio KItAgaWA* and Masako Yamaguchi*
}

(Received January 16, 1971)

\section{Introduction}

The object of this paper is to introduce a certain type of cellular automata, which are subject to a sequence of applications of local mapping transformations satisfying the principle of local majority, with an aim to formulate a mathematical model which may be verified to be useful as a basis of biomathematics. This paper is prepared to give the first step consideration to our approach plan suggested in the previous paper by one of us, Kitagawa [5].

In Section 2 we shall give some basic definitions regarding a finite, rectangular two-dimensional array of finite state automata, which is called an $m \times n$ cell space. A state of each cell in an $m \times n$ cell space is either 1 or 0 , and a configuration in an $m \times n$ cell space is an allocation of one of these two states 1 and 0 to each individual cell belonging to the $m \times n$ cell space. We shall introduce the notion of local mapping transformation satisfying the principle of local majority defined over each $2 \times 2$ cell space, which is called a $2 \times 2$ basic cell square. Such local mapping transformation is connected with the notion of firing point to be located in the central point of the $2 \times 2$ basic square. Each individual local mapping transformation will induce a transformation of configuration in the $m \times n$ cell space. In Section 3 we shall be concerned with the notion of firing points and with the process $\left\{X_{t}\right\}(t=0$, $1,2,3, \cdots)$ where $X_{t}$ denotes the set of firing points at the time point $t$. For an initial state $C_{0}$ the process $\left\{X_{t}\right\}$ will induce a transformation of configurations giving a sequence of the set of configurations $\left\{C_{t}\right\}(t=0,1,2,3, \cdots)$.

In Section 4 we shall investigate the behaviours of configuration transitions under somewhat resticted probabilistic scheme for which we are able to apply the formula well known in the theory of Markovian chain. In view of the considerations given in Section 4 it is remarkable that stable configurations under applications of local mapping transformation satisfying the principle of local majority have a certain simple pattern, as we show in Section 5 . The results so far obtained up to Section 5 are not completely satisfactory for us, in view of our original purpose to aim at some biomathematical model. There are in fact urgent needs for introducing

* Research Insititute of the Fundamental Information Science, Faculty of Science, Kyushu University, Fukuoka. 
a simple mathematical model in which some oscillatory phenomena can arise in its behaviours. In Section 6 we shall introduce an inhibition state $\phi$ and we shall show that some oscillatory phenomena will take place in our $m \times n$ cell space by our introduction of $\phi$ states. In view of some remarkable phenomena associated with introduction of $\phi$ states, we shall make further investigations on the roles and the functions of $\phi$ states in Section 7.

In summary of this introduction the authors should like to point out that the present paper is aimed to prepare the first step to information science approach to biomathematics and that more emphasises are placed upon an illustration of our fundamental ideas than upon formal theorem presentations.

It is also remarked that although we are appealing to some sort of cell space, our considerations to be developed in this paper are not concerned with any cellulars automaton theory hitherto developed by several authors such as Wang [10], Moore [7], von Neumann [9], Burks [1], Codd [2], and Minsky-Papert [6], but that we are here dealing with much simpler formulation upon which we want to build up some fundamental features of phenomena to be discussed in biomathematical approaches.

\section{Basic definitions regarding cell sapace}

Let us consider a finite, rectangular, two-dimensional iterative array of finite state automata. Such an array is pictured in Figure 1 with the automata represented by squares.

The lines connecting the squares represent communication channels between

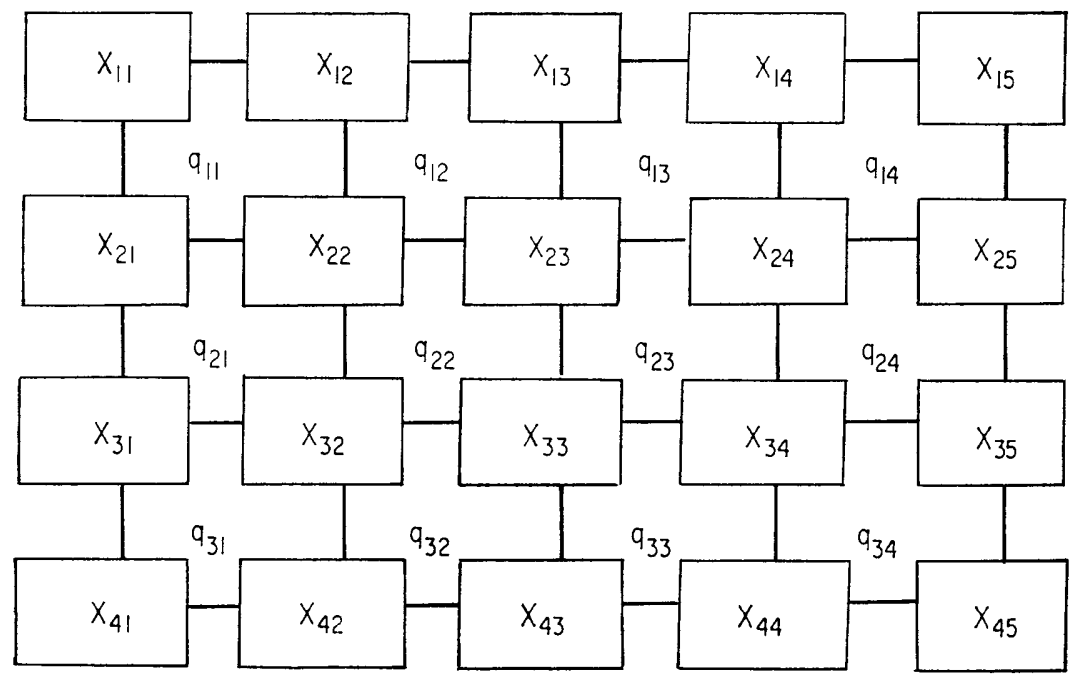

Fig. 1. $4 \times 5$ cell space.

$x_{i j}=$ the state of the $(i, j)$ cell.

$q_{i j}=$ the central point of the $2 \times 2$ basic cell space consisting of four cells located in $\mathrm{B}_{i j}:(i, j),(i, j+1),(i+1, j)$ and $(i+1, j+1)$. 
the automata.

The automata used in such a construction are called cells which are assumed to be isomorphic to each other. Each cell is assumed to be connected with its nearest neighbours whose number is four, three and two according to the situation that the cell is located in an interior, in a boundary and in a corner respectively. Let us call such a rectangular array of automata with $m$ rows $\times n$ columns to be an $m \times n$ cell space.

Let us assume that there are two alternatives, which are denoted by 1 and 0 respectively, regarding the possible states of each individual cell. A $2 \times 2$ square array of cells shown in Figure 2 is called to be a $2 \times 2$ basic cell square $B_{i j}$, where $x_{i j}$ denotes the state of the $(i, j)$ cell in the $2 \times 2$ basic square.

Thus a configuration of states in a $2 \times 2$ basic square can be denoted by a matrix

$$
\mathrm{M}_{i, j}^{(2,2)}(x)=\left(\begin{array}{ll}
x_{i j} & x_{i, j+1} \\
x_{i+1, j} & x_{i+1, j+1}
\end{array}\right)
$$

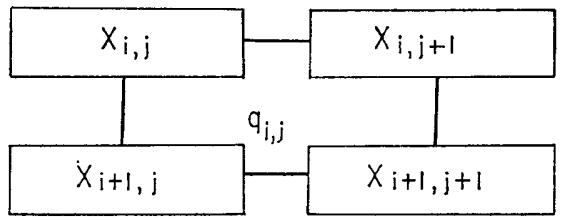

Fig. 2. Configuration $\mathrm{M}_{i j}^{(2,2)}(x)$ in a $2 \times 2$ basic cell square $\mathrm{B}_{i j}$.

An assignment of each state state $x_{i j}$ to each $(i, j)$ cell in an $m \times n$ cell space induces a configuration in this $m \times n$ cell space which can be denoted by a matrix

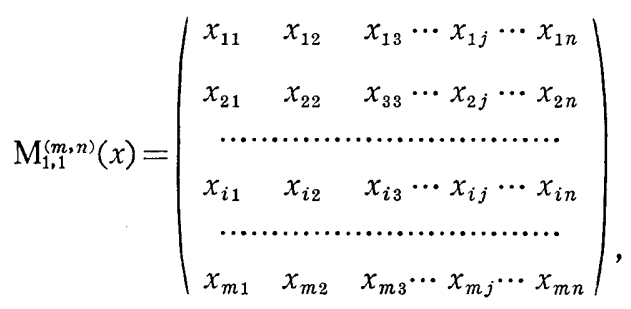

where each $x_{i j}$ is either one or zero. $\mathrm{M}_{1,1}^{(m, n)}(x)$ will be sometimes abbreviated by $\mathrm{M}^{(m, n)}(x)$.

Specially when all $m n x_{i j}$ turn out to be 1 , we shall denote $\mathrm{M}^{(m, n)}(x)$ by $\mathrm{I}^{(m, n)}$. Similarly when all $m n x_{i j}$ turn out to be zero, we shall denote $\mathrm{M}^{(m, n)}(x)$ by $\mathrm{O}^{(m, n)}$.

Particularly we have

$$
\mathrm{I}^{(2,2)}=\left(\begin{array}{ll}
1 & 1 \\
1 & 1
\end{array}\right), \quad \mathrm{O}^{(2,2)}=\left(\begin{array}{ll}
0 & 0 \\
0 & 0
\end{array}\right) .
$$

A transformation $\mathrm{T}$ of a configuration $\mathrm{M}^{(m, n)}(x)$ to another configuration $\mathrm{M}^{(m, n)}(y)$ is called a mapping transformation in the $m \times n$ cell space:

$$
\mathrm{T}: M^{(m, n)}(x) \longrightarrow \mathrm{M}^{(m, n)}(y) .
$$


A mapping transformation in a $2 \times 2$ cell space is called a local mapping transformation, which will be denoted by

$$
\text { LT }: \mathrm{M}^{(2,2)}(x) \longrightarrow \mathrm{M}^{(2,2)}(y) .
$$

In what follows we are particularly concerned with a certain type of local mapping transformation given by

Definition 1. A local mapping transformation $\mathrm{LT}: \mathrm{M}^{(2,2)}(x) \rightarrow \mathrm{M}^{(2,2)}(y)$ is said to satisfy the principle of local majority, if it is defined by the relation:

$$
\mathbf{M}_{i, j}^{(2,2)}(y)= \begin{cases}\mathrm{I}^{(2,2)}, & \text { if } S_{i j}(x)>2 \\ \mathrm{M}_{i j}^{(3,2)}(x), & \text { if } S_{i j}(x)=2 \\ 0^{(2,2)}, & \text { if } S_{i j}(x)<2,\end{cases}
$$

where we have put

$$
S_{i j}(x) \equiv \sum_{p=0}^{1} \sum_{q=0}^{1} x_{i+p, j+q} .
$$

In what follows we shall denote a local mapping transformation satisfying the principle of local majority by the abbreviated notation LMT (local majority transformation).

\section{Firing poins and applications of local mapping transformations}

One of the characteristic features of cellular automata is the fact that they can admit the possibility of simultaneous applications of certain local mapping transformation associated to each fundamental cell subspace, which may lead us to parallel computation procedures. This possibility may sometimes secure us parallel applications of all local mapping transformations associated with each $2 \times 2$ basic square in our $m \times n$ cell space.

However at our first step approach we should rather be concerned with a preparation of some more elementary notions in order to be able to discuss in the later stage some fundamental aspects of biological behaviours in large systems. In this sense we shall start with an introduction of the notion of firing points in this Section 3.

A firing of a $2 \times 2$ basic square means an application of LMT. Such a firing is assumed to be referred to the central point of each $2 \times 2$ basic square, which is henceforth called a firing point. Without any firing point, any $2 \times 2$ basic square remains unchanged, and with it in the central point the corresponding $2 \times 2$ basic square is subject to LMT.

Now it may be possible to define a parallel application of firing, as well as a sequential application of firing with reference to every $2 \times 2$ basic squares imbedded in an $m \times n$ cell space. However we shall begin with a survey of various procedures of firing instead of restricting our consideration within parallel computations. 


\section{Limiting behaviours of configuration transitions}

In this Section we shall be concerned with a stochastic process $\left\{X_{t}\right\}$ which is an idependent stochastic process with identical probability distribution (ISPI).

For each assigned initial configuration $C_{0}$, an application of firing sequence according to an ISPI $\left\{X_{t}\right\}$ will induce a Markovian stochastic process $\left\{C_{t}\right\}(t=0,1$, $2,3, \cdots)$ in such a way that

$$
\mathrm{T}_{t}:\left(C_{t}, X_{t+1}\right) \longrightarrow C_{t+1} .
$$

Let us denote by $p_{i j}$ the transition probability that the transition from one configuration $C_{i}$ to another configuration $C_{j}$. This transition from $C_{i}$ to $C_{j}$ is equivalent to the occurrance of the firing(s) of central point(s) $q$ for which the transition from a configuration $C_{i}$ to another configuration $C_{j}$ will be realised through an application of LMT to the $2 \times 2$ basic square having $q$ as its central point. Let us denote by $\mathrm{P} m \times n$ matrix of transition probabilities:

$$
\mathrm{P}=\left(p_{i, j}\right)
$$

and the $N$-th iteration of $P$ by

$$
\mathrm{P}^{(N)}=\left(p_{i j}^{(N)}\right) .
$$

Since the number of all the possible configurations in an $m \times n$ cell space under our formulation amounts to be $2^{m n}$, the matrix of the transition probabilities among these $2^{m n}$ configurations becomes to be a $2^{m n} \times 2^{m n}$ matrix.

However it can be easily seen that for each assigned initial configuration $C_{0}$ the sequence of configurations $\left\{C_{t}\right\}(t=0,1,2,3, \cdots)$ does not necessarily cover all the possible configurations but it is usually limited within some specific set of configuration depending upon the initial configuration $C_{0}$. This fact will make us possible to deal with a matrix $P$ whose order is comparatively small, because it is concerned with configurations belonging to $J\left(C_{0}\right)$ defined by :

$$
J\left(C_{0}\right)=\bigcup_{k=0}^{\infty} C_{k}
$$

for all possible sequences $\left\{C_{k}\right\}$ starting with the initial configuration $C_{0}$.

Now we are naturally interested with the transition behaviours among configurations belonging to $J\left(C_{0}\right)$ when we start with an initial configuration $C_{0}$ and also with their behavious as the number $N$ tends to infinity.

For this purpose there is a wellknown Theorem due to Romanosky [8] and Gantmacher [3] to the following effect.

THEOREM A (Romanonsky [8] and [3]). Let $\left(p_{i j}\right)(i, j=1,2, \cdots, k)$ be an $k \times k$ matrix of transition probabilities in the sense of Markovian chain.

Let the different characteristic roots of

$$
A(\lambda) \equiv\left|\begin{array}{cccc}
\lambda-p_{11} & -p_{12} & -p_{13} \cdots-p_{12 k} \\
-p_{21} & \lambda-p_{22} & -p_{23} \cdots-p_{22 k} \\
\cdots \cdots \cdots \cdots \cdots \cdots \cdots \cdots \cdots \cdots \cdots \\
-p_{k 1} & -p_{k 2} & -p_{k 3} \cdots \lambda-p_{k k}
\end{array}\right|=0
$$


be denoted by $\left\{\lambda_{i}\right\}(i=1,2, \cdots, \mu)$ with their respective multiplicies $m_{i}(i=1,2,3, \cdots, \mu)$.

Then we have, for each $N$, we have

$$
p_{\alpha \beta}^{(N)}=\sum_{i=0}^{\mu} \frac{1}{\left(m_{i}-1\right):} D_{\lambda}^{m_{i}-1}\left[\frac{\lambda^{N} A_{\alpha \beta}(\lambda)}{\phi_{i}(\lambda)}\right]_{\lambda=\lambda_{i}},
$$

where $A_{\alpha \beta}(\lambda)$ is the cofactor of the element $\lambda \delta_{\beta \alpha}-p_{\beta \alpha}$ in the determinant of $A(\lambda)$ and $\psi_{i}(\lambda)$ is defined by

$$
A(\lambda) \equiv\left(\lambda-\lambda_{i}\right)^{m_{i}} \phi_{i}(\lambda), \quad(i=1,2, \cdots, \mu),
$$

while $D_{\lambda}^{m_{i}-1}$ denotes the $\left(m_{i}-1\right)$ times differentiation with respect to $\lambda$.

The extensive applications of this theorem are given in Kitagawa [4] in connection with successive process of statistical controls. In our present formulation of cellular automata an application will be quite useful for investigation of the behaviours both at each assigned finite stage and in the limiting case when $N$ becomes infinity. We shall now give two examples which will be sufficient enough how far and why such an application of THEOREM $A$ is helpful for our purpose.

EXAMPLE 1. In a $3 \times 3$ cell space let us consider an initial configuration

$$
C_{0}=\left(\begin{array}{lll}
0 & 1 & 1 \\
0 & 1 & 1 \\
0 & 0 & 0
\end{array}\right) .
$$

Then the other configurations belonging to the set $J\left(C_{0}\right)$ of all configurations induced by applications of LMT is given by

$$
\begin{aligned}
& C_{1}=\left(\begin{array}{lll}
0 & 1 & 1 \\
0 & 0 & 1 \\
0 & 0 & 0
\end{array}\right), \quad C_{2}=\left(\begin{array}{lll}
0 & 1 & 1 \\
0 & 0 & 0 \\
0 & 0 & 0
\end{array}\right) \text {, } \\
& C_{3}=\left(\begin{array}{lll}
0 & 0 & 1 \\
0 & 0 & 1 \\
0 & 0 & 0
\end{array}\right), \quad C_{4}=\left(\begin{array}{lll}
0 & 0 & 1 \\
0 & 0 & 0 \\
0 & 0 & 0
\end{array}\right), \\
& C_{5}=\left(\begin{array}{lll}
0 & 0 & 0 \\
0 & 0 & 0 \\
0 & 0 & 0
\end{array}\right)
\end{aligned}
$$

The transition matrix $\mathrm{P}$ has the form 


$$
\begin{aligned}
& \begin{array}{llllll}
C_{0} & C_{1} & C_{2} & C_{3} & C_{4} & C_{5}
\end{array} \\
& \left.\mathrm{P}=\begin{array}{l|llllll}
C_{2} & p_{11} & p_{12} & 0 & 0 & 0 & 0 \\
C_{1} & p_{21} & p_{22} & p_{23} & p_{24} & 0 & 0 \\
C_{3} & 0 & 0 & p_{33} & 0 & p_{35} & 0 \\
C_{4} & 0 & 0 & p_{44} & p_{45} & 0 \\
C_{5} & 0 & 0 & 0 & 0 & p_{55} & p_{56} \\
0 & 0 & 0 & 1
\end{array}\right) .
\end{aligned}
$$

The connection routes among these six configurations are given by Figure 3.

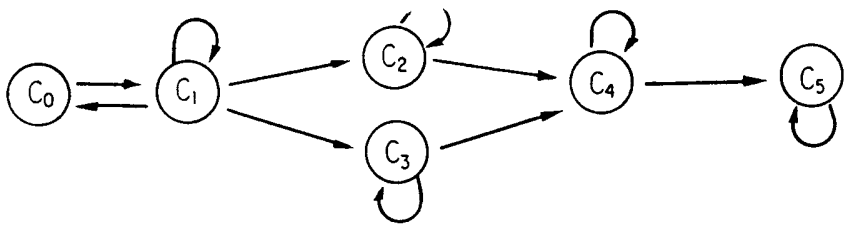

Fig. 3. Connection routes among configurations in Example 1.

The characteristic equation becomes

$$
A(\lambda)=(\lambda-1)\left(\lambda-p_{3,3}\right)\left(\lambda-p_{4,4}\right)\left(\lambda-p_{5,5}\right) Q_{2}(\lambda)=0,
$$

where we have put

$$
\begin{aligned}
Q_{2}(\lambda) & =\lambda^{2}-\left(p_{1,1}+p_{2,2}\right) \lambda+p_{1,1} p_{2,2}-p_{1,2} p_{2,1} \\
& =(\lambda-\alpha)(\lambda-\beta), \text { say. }
\end{aligned}
$$

For the sake of illustration, let us now consider the special case when

$$
\text { Pr. }\left\{X_{t}=q_{i, j}\right\}=\frac{1}{4}
$$

for $i, j=1,2$, that is, the case of the equal probability of firing for each of the four central points. In this case the transition matrix $P$ becomes

$$
\mathrm{P}=\left(\begin{array}{cccccc}
\frac{3}{4} & \frac{1}{4} & 0 & 0 & 0 & 0 \\
\frac{1}{4} & \frac{1}{4} & \frac{1}{4} & \frac{1}{4} & 0 & 0 \\
0 & 0 & \frac{3}{4} & 0 & \frac{1}{4} & 0 \\
0 & 0 & 0 & \frac{3}{4} & \frac{1}{4} & 0 \\
0 & 0 & 0 & 0 & \frac{3}{4} & \frac{1}{4} \\
0 & 0 & 0 & 0 & 0 & 1
\end{array}\right),
$$

and hence we have 


$$
\begin{aligned}
A(\lambda) & =(\lambda-1)\left(\lambda-\frac{3}{4}\right)^{3}\left(\lambda^{2}-\lambda+\frac{1}{8}\right) \\
& =(\lambda-1)\left(\lambda-\frac{3}{4}\right)^{3}(\lambda-\alpha)(\lambda-\beta), \text { say. }
\end{aligned}
$$

Now the application of THEOREM A yields us

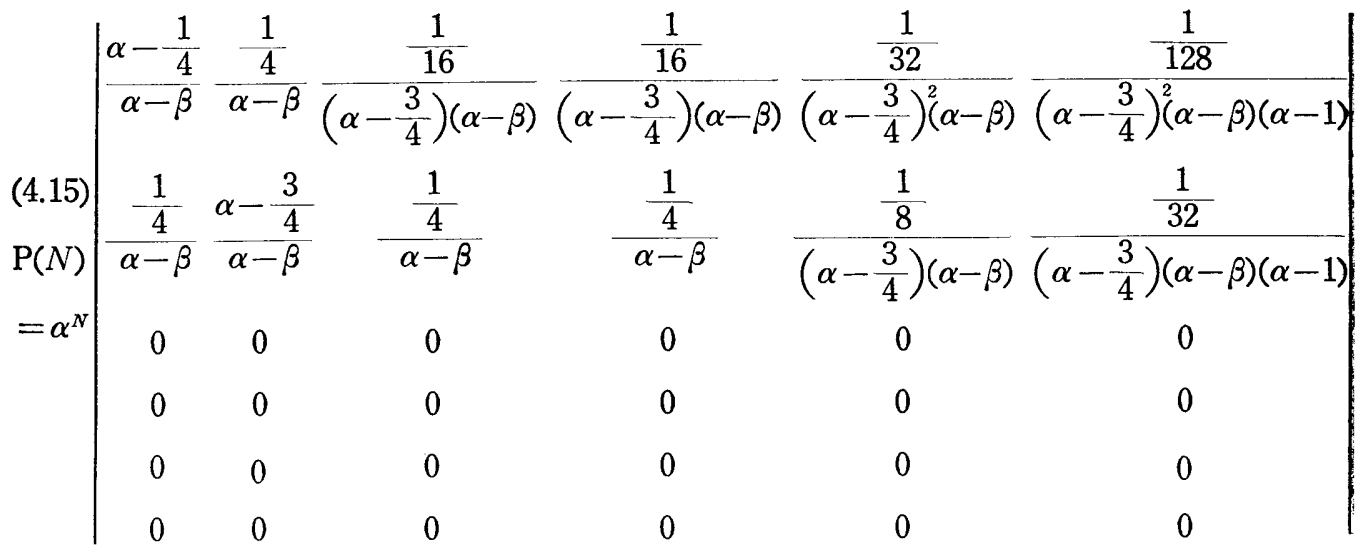

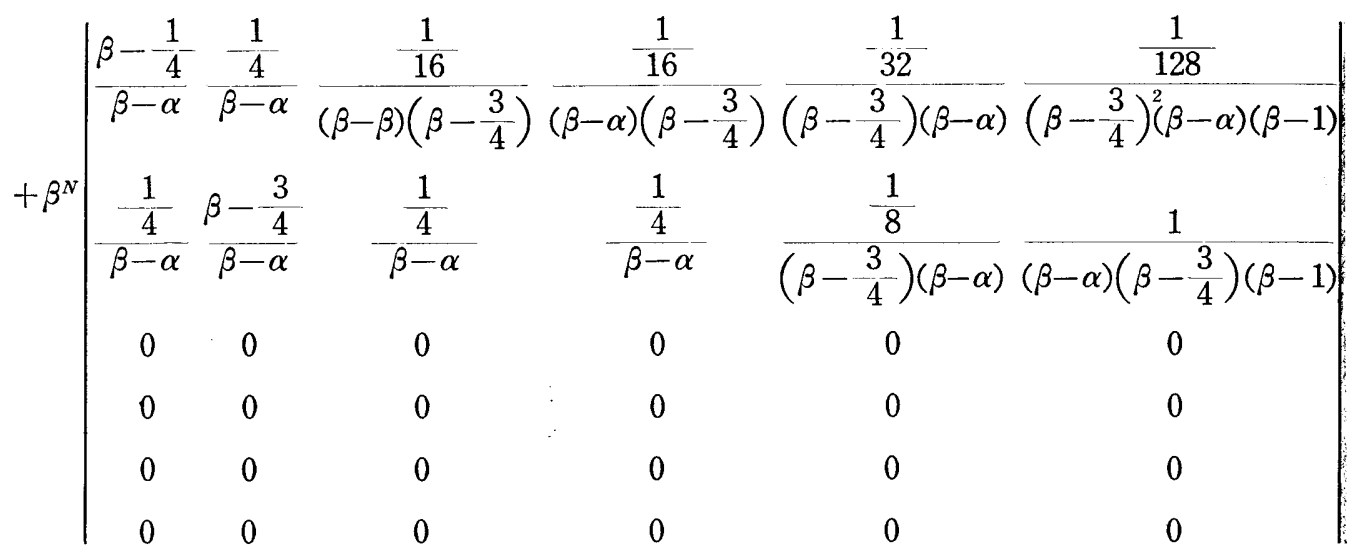

$$
\begin{aligned}
& +1^{N}\left|\begin{array}{cccccc}
0 & 0 & 0 & 0 & 0 & 1 \\
0 & 0 & 0 & 0 & 0 & 1 \\
0 & 0 & 0 & 0 & 0 & 1 \\
0 & 0 & 0 & 0 & 0 & 1 \\
0 & 0 & 0 & 0 & 0 & 1 \\
0 & 0 & 0 & 0 & 0 & 1
\end{array}\right|
\end{aligned}
$$




$$
+\left(\frac{3}{4}\right)^{N}\left|\begin{array}{cccccc}
0 & 0 & -1 & -1 & -2-N-4 & \frac{4}{3} N+12 \\
0 & 0 & 0 & 0 & -2 & 2 \\
0 & 0 & 1 & 0 & -\frac{N}{3} & -\left(1+\frac{N}{3}\right) \\
0 & 0 & 0 & 1 & -\frac{N}{3} & -\left(1+\frac{N}{3}\right) \\
0 & 0 & 0 & 0 & 1 & -1 \\
0 & 0 & 0 & 0 & 0 & 0
\end{array}\right|
$$

In the consequence we have

$$
\mathrm{P}^{(\infty)}=\left(p_{i j}^{(\infty)}\right)=\left(\begin{array}{llllll}
0 & 0 & 0 & 0 & 0 & 1 \\
0 & 0 & 0 & 0 & 0 & 1 \\
0 & 0 & 0 & 0 & 0 & 1 \\
0 & 0 & 0 & 0 & 0 & 1 \\
0 & 0 & 0 & 0 & 0 & 1 \\
0 & 0 & 0 & 0 & 0 & 1
\end{array}\right),
$$

which shows that the configuration $C_{5}$ is the unique absorbing state in the theory of Markovian chain.

EXAMPLE 2. In a $3 \times 3$ cell space, let us consider an initial configuration

$$
C_{0}=\left(\begin{array}{lll}
0 & 1 & 1 \\
1 & 0 & 1 \\
1 & 0 & 0
\end{array}\right)
$$

Thenjthe other configurations belonging to the set of configuration $J\left(C_{0}\right)$ are given by the following eleven ones:

$$
\begin{array}{ll}
C_{1}=\left(\begin{array}{lll}
0 & 0 & 1 \\
0 & 0 & 1 \\
1 & 0 & 0
\end{array}\right), \quad C_{2}=\left(\begin{array}{lll}
0 & 1 & 1 \\
0 & 1 & 1 \\
1 & 0 & 0
\end{array}\right), \quad C_{3}=\left(\begin{array}{lll}
0 & 1 & 1 \\
0 & 0 & 0 \\
1 & 0 & 0
\end{array}\right) \\
C_{4}=\left(\begin{array}{lll}
0 & 1 & 1 \\
0 & 0 & 1 \\
0 & 0 & 0
\end{array}\right), \quad C_{5}=\left(\begin{array}{lll}
0 & 0 & 1 \\
0 & 0 & 0 \\
1 & 0 & 0
\end{array}\right), \quad C_{6}=\left(\begin{array}{lll}
0 & 0 & 1 \\
0 & 0 & 1 \\
0 & 0 & 0
\end{array}\right) \\
C_{7}=\left(\begin{array}{lll}
0 & 1 & 1 \\
0 & 0 & 0 \\
0 & 0 & 0
\end{array}\right), \quad C_{8}=\left(\begin{array}{lll}
0 & 1 & 1 \\
0 & 1 & 1 \\
0 & 0 & 0
\end{array}\right), \quad C_{9}=\left(\begin{array}{lll}
0 & 0 & 0 \\
0 & 0 & 0 \\
1 & 0 & 0
\end{array}\right)
\end{array}
$$




$$
C_{10}=\left(\begin{array}{lll}
0 & 0 & 1 \\
0 & 0 & 0 \\
0 & 0 & 0
\end{array}\right), \quad C_{11}=\left(\begin{array}{lll}
0 & 0 & 0 \\
0 & 0 & 0 \\
0 & 0 & 0
\end{array}\right) .
$$

The connection routes among these twelve configurations are given by Figure 4 .

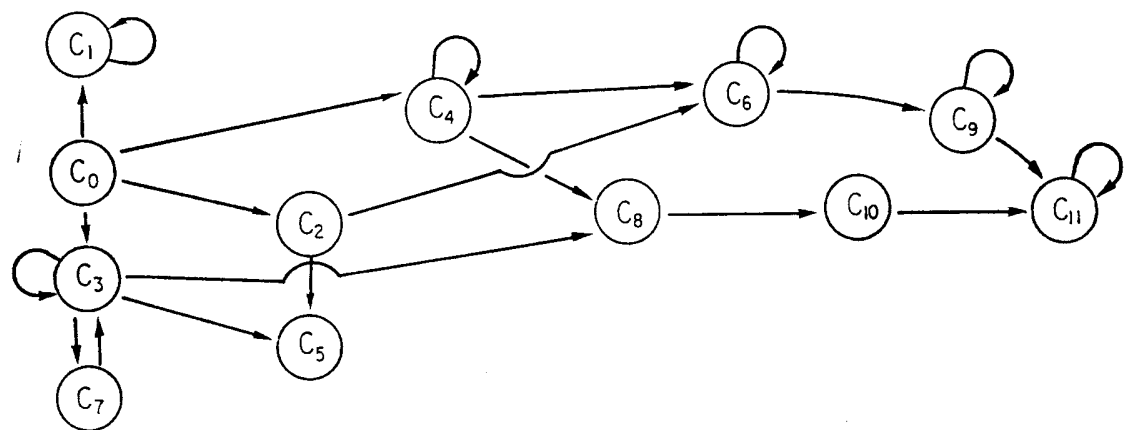

Fig. 4. Connection routes among configurations in Example 2.

The transition matrix $P$ can be obtained similarly as in Example 1. The characteristic equation becomes

$$
\begin{aligned}
A(\lambda)= & \lambda(\lambda-1)^{2}\left(\lambda-p_{3,3}\right)\left(\lambda-p_{5,5}\right)\left(\lambda-p_{6,6}\right) \\
& \times\left(\lambda-p_{7,7}\right)\left(\lambda-p_{9,9}\right)\left(\lambda-p_{10,10}\right)\left(\lambda-p_{11,11}\right) \\
& \times Q_{2}(\lambda)=0,
\end{aligned}
$$

where we have put

$$
Q_{2}(\lambda)=\left|\begin{array}{cc}
\lambda-p_{4,4} & -p_{4,8} \\
-p_{8,4} & \lambda-p_{8,8}
\end{array}\right| .
$$

For the special case of the equal probability of firing for each of the four central points $\left\{q_{i j}\right\}(i, j=1,2)$, that is, when (4.13) hold true, the transition matrix $P$ has the specific values such as

$$
\left\{\begin{array}{l}
p_{3,3}=p_{5,5}=p_{7,7}=\frac{1}{2} \\
p_{4,4}=p_{8,4}=p_{4,8}=\frac{1}{4} \\
p_{6,6}=p_{8,8}=p_{9,9}=p_{10,10}=p_{11,11}=3
\end{array}\right.
$$

and hence the characteristic equation becomes

$$
A(\lambda)=(\lambda-1)^{2} \lambda\left(\lambda-\frac{1}{2}\right)^{3}\left(\lambda-\frac{3}{4}\right)^{4} Q_{2}(\lambda)=0
$$

with $Q_{2}(\lambda)=\lambda^{2}-\lambda+\left(\begin{array}{c}1 \\ -8\end{array}\right)$.

In this specific EXAMPLE there are two specific configurations $C_{1}$ and $C_{11}$ each of which is an absorbing state in the theory of Markovian chain.

The application of THEOREM $\mathrm{A}$ is useful for dealing with this ExAMPLE. For 
instance, in order to calculate the asymptotic value of $p_{1,2}^{(N)}$ for large $N$ by virtue of

$$
p_{1,12}^{(N)}=\sum_{i=1}^{\mu} \frac{1}{\left(m_{i}-1\right)} D_{\lambda}^{m_{i}-1}\left[\frac{\lambda^{N} A_{1,12}(\lambda)}{\phi_{i}(\lambda)}\right]_{\lambda=\lambda_{i}},
$$

we can concentrate our attention to the root $\lambda_{1}=1$ with the multiplicity $m_{1}=2$. The direct calculation gives us

$$
\left(\frac{d}{d \lambda} A_{1,2}(\lambda)\right)_{\lambda=1}=\frac{3}{4}-\left(\frac{1}{4}\right)^{4}\left(\frac{1}{2}\right)^{3} Q_{2}(1)
$$

which gives

and hence

$$
D_{\lambda}^{(1)}\left[\frac{\lambda^{N} A_{1,12}(\lambda)}{\phi_{1}(\lambda)}\right]_{\lambda=1}=\frac{3}{4}
$$

$$
\lim _{N \rightarrow \infty} p_{1,12}^{(N)}=\frac{3}{4}
$$

\section{Stable configuration}

This Section 5 is a continuation to the previous Section 4 in the sense that our discussions here will be entirely based upon and referred to the same mathematical formulation of independent stochastic process with the identical probability distribution ISPI as they were in Section 4. Under the present circumstance some results can be obtained by appealing to the theory of Markovian chains, as we have shown in the applications of THEOREM $A$ in the previous Section 4. In fact, theoretically speaking, there is no reason why we cannot generalise some specific results to a general $m \times n$ cell space, except an unfavourable fact that the complexity of computations will increase very fast as $m$ and/or $n$ increase.

Indeed we can not deny that the complexity of computations being involved for persuiting our plan becomes more and more and even formidable to perform our plan as $m$ and/or $n$ increase indefinitely. It is therefore indispensable to introduce some crucial notions which are connected with asymptotic behaviour of the cell space. In fact, among $2^{m n}$ possible configurations in an $m \times n$ cell space, there does exist a certain subset of configurations, that is, stable configurations, which will be defined as follows :

Definition 2. A configuration $C$ in an $m \times n$ cell space, is said to be stable, if for any assigned configuration $C^{\prime}$ which is different from $C$, the probability of occurance of the central firing points which induces a mapping transformation from the configuration $C$ to $C^{\prime}$ is equal to zero, that is to say,

$$
\operatorname{Pr} .\left\{q / q: C \rightarrow C^{\prime}\right\}=0,
$$

which implies and is implied by

$$
\operatorname{Pr} .\{q / q: C \rightarrow C\}=1 .
$$

We have the following fundamental

THEOREM 1. In an $m \times n$ cell space the number of all the possible stable configurations is $2^{n+m-1}$. 
Proof. Let us prove the assertion of THEOREM 1 by induction. For $m=1$, any configuration is stable, because no application of LMT is possible for a $1 \times n$ cell space.

Hence the number of all stable configurations for a $1 \times n$ cell space is equal to $2^{n}=2^{1+n-1}$.

Let us assume that the assertion is valid for $m=r$, and let us prove that it holds for $m=r+1$.

Let a stable configuration in the $r \times n$ cell space be given by

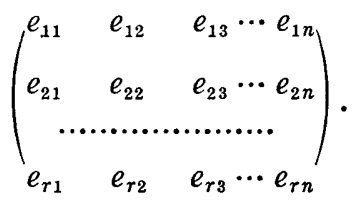

For the set $\left(e_{r 1}, e_{r_{2}}\right)$ there are the four alternatives such as $(0,0),(0,1),(1,0)$ and $(1,1)$. To each individual alternative there are two alternatives each of which is stable under the application of LMT. For example for the case when $\left(e_{r_{1}}=0, e_{r_{2}}=0\right)$, there are two alternatives $\left(e_{r+1,1}=0, e_{r+1,2}=0\right)$ and $\left(e_{r+1,1}=1, e_{r+1,2}=1\right)$, which will be denoted by

$$
\left(\begin{array}{ll}
0 & 0 \\
0 & 0
\end{array}\right), \quad\left(\begin{array}{ll}
0 & 0 \\
1 & 1
\end{array}\right) .
$$

In this way all the possible configurations are given by the following eight $2 \times 2$ basic squares:

$$
\begin{aligned}
& \left(\begin{array}{ll}
0 & 0 \\
0 & 0
\end{array}\right),\left(\begin{array}{ll}
0 & 0 \\
1 & 1
\end{array}\right),\left(\begin{array}{ll}
0 & 1 \\
0 & 1
\end{array}\right),\left(\begin{array}{ll}
0 & 1 \\
1 & 0
\end{array}\right),\left(\begin{array}{ll}
1 & 0 \\
0 & 1
\end{array}\right), \\
& \left(\begin{array}{ll}
1 & 0 \\
1 & 0
\end{array}\right),\left(\begin{array}{ll}
1 & 1 \\
0 & 0
\end{array}\right),\left(\begin{array}{ll}
1 & 1 \\
1 & 1
\end{array}\right) .
\end{aligned}
$$

Now let us consider the $2 \times 2$ basic squares defined by

$$
\left(\begin{array}{ll}
e_{r, 2} & e_{r, 3} \\
e_{r+1,2} & *
\end{array}\right)
$$

where $e_{r, 2}, e_{r, 3}$ and $e_{r+1,2}$ have already been defined.

There is exactly one determination of the value $e_{r+1,3}$ by which the $2 \times 2$ square become stable. Continuing in this way we shall observe that $\left\{e_{r+1, j}\right\} j \geqq 4$ will be vniquely determined. Therefore there are exactly two possible sets of value $\left\{e_{r+1, j}\right\}$ $(j=1,2, \cdots, n)$. Consequently we obtain the result that the assertion holds true for $m=r+1$ in the sense that there are exactly $2 \times 2^{r+n-1}=2^{(r+1)+n-1}$ stable configurations. This completes the induction.

It is useful to introduce

Definition 3. A configuration $C$ in an $m \times n$ cell space is said to be complementary to another configuration $C^{\prime}$ when for every pair $(i, j), 1 \leqq i \leqq m, 1 \leqq j \leqq n$, the $(i, j)$ elements $x_{i j}(C)$ of $C$ is conjugate to the corresponding element $x_{i j}\left(C^{\prime}\right)$ of $C^{\prime}$ in the 
sense that $x_{i j}\left(C^{\prime}\right)=1-x_{i j}(C)=\bar{x}_{i j}(C)$, say.

THEOREM 2. A configuration which is complementary to a stable configuration is stable.

The proof is trivial, because it does hold true for any LMT in the case of $2 \times 2$ square.

EXAMPLE 3. For a $3 \times 3$ cell space, there are $2^{3+3-1}=2^{5}=32$ stable configurations, which are given by the following 16 configurations and their respective complementary configurations.

$$
\begin{aligned}
& \left(\begin{array}{lll}
1 & 1 & 1 \\
0 & 0 & 0 \\
0 & 0 & 0
\end{array}\right) \quad\left(\begin{array}{lll}
0 & 0 & 0 \\
1 & 1 & 1 \\
0 & 0 & 0
\end{array}\right) \quad\left(\begin{array}{lll}
0 & 0 & 0 \\
0 & 0 & 0 \\
1 & 1 & 1
\end{array}\right) \\
& \left(\begin{array}{lll}
1 & 0 & 0 \\
1 & 0 & 0 \\
1 & 0 & 0
\end{array}\right) \quad\left(\begin{array}{lll}
0 & 1 & 0 \\
0 & 1 & 0 \\
0 & 1 & 0
\end{array}\right) \quad\left(\begin{array}{lll}
0 & 0 & 1 \\
0 & 0 & 1 \\
0 & 0 & 1
\end{array}\right) \\
& \left(\begin{array}{lll}
1 & 1 & 0 \\
0 & 0 & 1 \\
0 & 0 & 1
\end{array}\right) \quad\left(\begin{array}{lll}
1 & 0 & 1 \\
0 & 1 & 0 \\
0 & 1 & 0
\end{array}\right) \quad\left(\begin{array}{lll}
1 & 0 & 0 \\
0 & 1 & 1 \\
1 & 0 & 0
\end{array}\right) \\
& \left(\begin{array}{lll}
0 & 1 & 1 \\
1 & 0 & 0 \\
1 & 0 & 0
\end{array}\right) \quad\left(\begin{array}{lll}
0 & 1 & 0 \\
1 & 0 & 1 \\
0 & 1 & 0
\end{array}\right) \quad\left(\begin{array}{lll}
0 & 0 & 1 \\
1 & 1 & 0 \\
0 & 0 & 1
\end{array}\right) \\
& \left(\begin{array}{lll}
0 & 0 & 1 \\
0 & 0 & 1 \\
1 & 1 & 0
\end{array}\right) \quad\left(\begin{array}{lll}
0 & 1 & 0 \\
0 & 1 & 0 \\
1 & 0 & 1
\end{array}\right) \quad\left(\begin{array}{lll}
1 & 0 & 0 \\
1 & 0 & 0 \\
0 & 1 & 1
\end{array}\right) \\
& \left(\begin{array}{lll}
0 & 0 & 0 \\
0 & 0 & 0 \\
0 & 0 & 0
\end{array}\right)
\end{aligned}
$$

It is now easy to observe

THEOREM 3. A stable configuration in an $m \times n$ cell space has the following pattern:

$\left(1^{\circ}\right)$ There is a pair of partitions such that

$$
\begin{aligned}
& m=m_{1}+m_{2}+\cdots+m_{k} \\
& n=n_{1}+n_{2}+\cdots+n_{l},
\end{aligned}
$$

with positive integers $m_{1}$ and $n_{j}, 1 \leqq i \leqq k, 1 \leqq j \leqq l$ where $k$ and $l$ are subject to

$$
1 \leqq k \leqq m, \quad 1 \leqq l \leqq n
$$


$\left(2^{\circ}\right)$ In correspondence with the pair of partitions given in $\left(1^{\circ}\right)$, the whole $m \times n$ cell space is divided in $k l$ subspaces, each of which will be denoted by $S\left(m_{i}, n_{j}\right)(i=1$, $2, \cdots, k ; j=1,2, \cdots, l)$.

$\left(3^{\circ}\right)$ (a) The elements of each subspace $S\left(m_{i}, n_{j}\right)$ are entirely either 1 or zero.

A subspace $S\left(m_{i}, n_{j}\right)$ all of whose elements are equal to 1 is called to be of type I, while a subspace all of whose elements are equal to zero to be of type 0.

(b) Subspaces of these two types occur alternatively in an $m \times n$ cell space (See Figure 5).

\begin{tabular}{l|c|c|c|c|c|c|} 
& $n_{1}$ & $n_{2}$ & $n_{3}$ & $n_{4}$ & $n_{5}$ & $n_{6}$ \\
\hline$m_{1}$ & $I$ & 0 & $I$ & 0 & $I$ & 0 \\
\hline$m_{2}$ & 0 & $I$ & 0 & $I$ & 0 & $I$ \\
\hline$m_{3}$ & $I$ & 0 & $I$ & 0 & $I$ & 0 \\
$m_{4}$ & 0 & $I$ & 0 & $I$ & 0 & $I$
\end{tabular}

Fig. 5. Pattern of stable configuration.

\section{Introduction of inhibition state $\phi$}

According to the results which we have obtained in the previous Sections 2-5 there have been shown an existence of stable configurations to which the applications of LMT will converge.

Although the results may have some interests in themselves we can not be completely satisfory in view of our original purpose to aim at some biomathematical model. There are in fact urgent needs for introducing a simple mathematical medel in which some oscillatory phenomena can arise in its behaviours. The purpose of this Section is to introduce an inhibition state $\phi$ which secures a certain set of oscillatory phenomena by giving some restrictions to the free application of LMT.

DEFINITION 4. A state $\phi$ to be attached to each cell is called to be an inhibitation state in the sense that $2 \times 2$ basic cell square having at least one cell whose state is $\phi$ is invariant under any any LMT.

An introduction of inhibitation states in an $m \times n$ space has the possibility of the following characteristic phenomena which have been not yet observed in the preceeding Section of this paper. Let us begin with the following ExAMPLES.

EXAMPLE 4. Let us consider an initial configuration

$$
C=\left(\begin{array}{lll}
\phi & 0 & 0 \\
1 & 1 & 0 \\
1 & 1 & \phi
\end{array}\right) .
$$

The possible positions of central points at which firing may take place are four 
as we have explained previous.

However the existence of two $\phi$ 's gives us two $2 \times 2$ basic squares each of which is invariant.

Consequently there is only one other possible configuration

$$
C_{1}=\left(\begin{array}{lll}
\phi & 0 & 0 \\
1 & 0 & 0 \\
1 & 1 & \phi
\end{array}\right)
$$

The connection route between these two confiuration is simply given by Figure 6 .

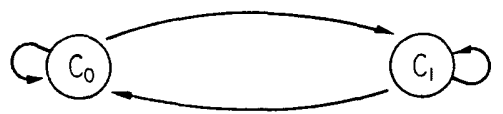

Fig. 6. Oscillation between $C_{0}$ and $C_{1}$.

In fact there are two active central points $q_{1,2}$ and $q_{2,1}$ for firing. Under the assumption of equal probability for each of these two firing points, the characteristic equation of the transition matrix between two configurations $C_{0}$ and $C_{1}$ is given by

$$
A(\lambda) \equiv\left|\begin{array}{lr}
X-1 & 1 \\
2 & 2 \\
-1 & \lambda-\frac{1}{2}
\end{array}\right|=\lambda(1-1)=0 .
$$

EXAMPLE 5. Let us consider a configuration $C_{0}$ in a $3 \times 4$ cell space given by

$$
C_{0}=\left(\begin{array}{llll}
\phi & 0 & 0 & 0 \\
1 & 0 & 0 & 0 \\
1 & 1 & 1 & \phi
\end{array}\right)
$$

There are two other configurations $C_{1}$ and $C_{2}$ connected with ${ }_{2}^{\top} C_{0}$ by the transition rule which is based upon the principle of local majority.

$$
C_{1}=\left(\begin{array}{llll}
\phi & 0 & 0 & 0 \\
1 & 1 & 0 & 0 \\
1 & 1 & 1 & \phi
\end{array}\right) \quad C_{2}=\left(\begin{array}{llll}
\phi & 0 & 0 & 0 \\
1 & 1 & 1 & 0 \\
1 & 1 & 1 & \phi
\end{array}\right)
$$

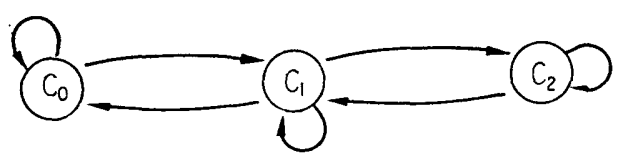

Fig. 7. Oscillations among $C_{0}, C_{1}$ and $C_{2}$.

Now the matrix of transition probabilities among these three configurations are given by a $3 \times 3$ matrix $\left(p_{i j}\right)$, where

$$
p_{i j}=\operatorname{Pr} .\left\{q \mid q: C_{i} \rightarrow C_{j}\right\},
$$


for $i, j=0,1,2$.

There are four active central points $q_{1,2}, q_{1,3}, q_{2,1}$ and $q_{2,2}$.

The assumption of equal probability for each of these four active central points gives us the characteristic equation

$$
\begin{aligned}
A(\lambda) & =\left|\begin{array}{ccc}
\lambda-\frac{3}{4} & -\frac{1}{4} & 0 \\
-\frac{1}{4} & \lambda-\frac{1}{2} & -\frac{1}{4} \\
0 & -\frac{1}{4} & \lambda-\frac{3}{4}
\end{array}\right| \\
& =(\lambda-1)\left(\lambda-\frac{3}{4}\right)\left(\lambda-\frac{1}{4}\right)=0 .
\end{aligned}
$$

The matrix of transition probabilities at the $N$-th step is given by

$$
\begin{aligned}
\mathrm{P}^{(N)}= & \frac{1}{3}\left[\begin{array}{lll}
1 & 1 & 1 \\
1 & 1 & 1 \\
1 & 1 & 1
\end{array}\right]+\left(\frac{1}{2}\right)\left(\frac{3}{4}\right)^{N}\left[\begin{array}{rrr}
1 & 0 & -1 \\
0 & 0 & 0 \\
-1 & 0 & 1
\end{array}\right] \\
& +\frac{1}{6}\left(-\frac{1}{4}\right)^{N}\left[\begin{array}{rrr}
1 & -2 & 1 \\
-2 & 4 & -2 \\
1 & -2 & 1
\end{array}\right] .
\end{aligned}
$$

EXAmple 6. An initial configuration $C_{0}$ is defined in a $5 \times 4$ cell space in the following form.

$$
C_{0}=\left(\begin{array}{lllll}
\phi & 0 & 0 & 0 & 0 \\
1 & 0 & 0 & 0 & 0 \\
1 & 0 & 0 & 0 & 0 \\
1 & 1 & 1 & 1 & \phi
\end{array}\right) .
$$

There are now eight other configurations $C_{i}(i=1,2,3,4,5,6,7,8)$ connected with $C_{0}$ by the transition rule which is based upon the principle of local majority.

$$
\begin{aligned}
& C_{1}=\left(\begin{array}{lllll}
\phi & 0 & 0 & 0 & 0 \\
1 & 0 & 0 & 0 & 0 \\
1 & 1 & 0 & 0 & 0 \\
1 & 1 & 1 & 1 & \phi
\end{array}\right) \quad C_{2}=\left(\begin{array}{lllll}
\phi & 0 & 0 & 0 & 0 \\
1 & 1 & 0 & 0 & 0 \\
1 & 1 & 0 & 0 & 0 \\
1 & 1 & 1 & 1 & \phi
\end{array}\right) \\
& C_{3}=\left(\begin{array}{lllll}
\phi & 0 & 0 & 0 & 0 \\
1 & 0 & 0 & 0 & 0 \\
1 & 1 & 1 & 0 & 0 \\
1 & 1 & 1 & 1 & \phi
\end{array}\right) \quad C_{4}=\left(\begin{array}{lllll}
\phi & 0 & 0 & 0 & 0 \\
1 & 1 & 0 & 0 & 0 \\
1 & 1 & 1 & 0 & 0 \\
1 & 1 & 1 & 1 & \phi
\end{array}\right)
\end{aligned}
$$




$$
\begin{array}{rlrl}
C_{5} & =\left(\begin{array}{lllll}
\phi & 0 & 0 & 0 & 0 \\
1 & 1 & 1 & 0 & 0 \\
1 & 1 & 1 & 0 & 0 \\
1 & 1 & 1 & 1 & \phi
\end{array}\right) & C_{6}=\left(\begin{array}{llllll}
\phi & 0 & 0 & 0 & 0 \\
1 & 1 & 0 & 0 & 0 \\
1 & 1 & 1 & 1 & 0 \\
1 & 1 & 1 & 1 & \phi
\end{array}\right) \\
C_{7}=\left(\begin{array}{lllll}
\phi & 0 & 0 & 0 & 0 \\
1 & 1 & 1 & 0 & 0 \\
1 & 1 & 1 & 1 & 0 \\
1 & 1 & 1 & 1 & \phi
\end{array}\right) & C_{8}=\left(\begin{array}{lllll}
\phi & 0 & 0 & 0 & 0 \\
1 & 1 & 1 & 1 & 0 \\
1 & 1 & 1 & 1 & 0 \\
1 & 1 & 1 & 1 & \phi
\end{array}\right)
\end{array}
$$

The connection route among these nine configurations are given by Figure 8 .

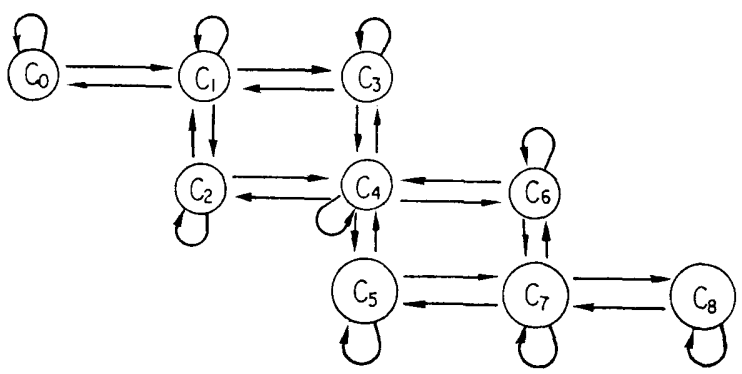

Fig. 8. Oscillations among nine configurations $\left\{C_{i}\right\}(i=0,1,2, \cdots, 8)$.

\section{Roles and functions of $\phi$-state}

There are several important applications of the functions of $\phi$ states.

(a) PARTition OF CELl SPACE INTO A SET OF MUTUALly INDEPENDENT CELL SUBSPACES

A certain configuration of $\phi$ states in an $m \times n$ cell space with a certain set of peripherical segments can give us a partition of the $m \times n$ cell space into a set of cell subspaces which are mutually independent with other subspaces in the in the sense that each of these subspaces will show a pattern of oscillatory change of configurations independently.

A contruction of such a partition of an $m \times n$ cell space is obtained by virtue of THEOREM 4 just mentioned.

The situation can be easily illustrated by the following.

EXAMPLE 7 . Let us consider a $16 \times 15$ cell square with a configuration given in Figure 9.

There are twelve cell subspaces A, B, C, D, E, F, G, H, I, J, K and L. Each of them shows its ergodic oscillatory changes among a certain set of configurations without any mutual interdependence with other subspaces. This is a picture of selfconservations of each subspace which are secured by the allocation pattern of $\phi$ states and the assignments of 1 and 0 as boundaries among these subspaces. 


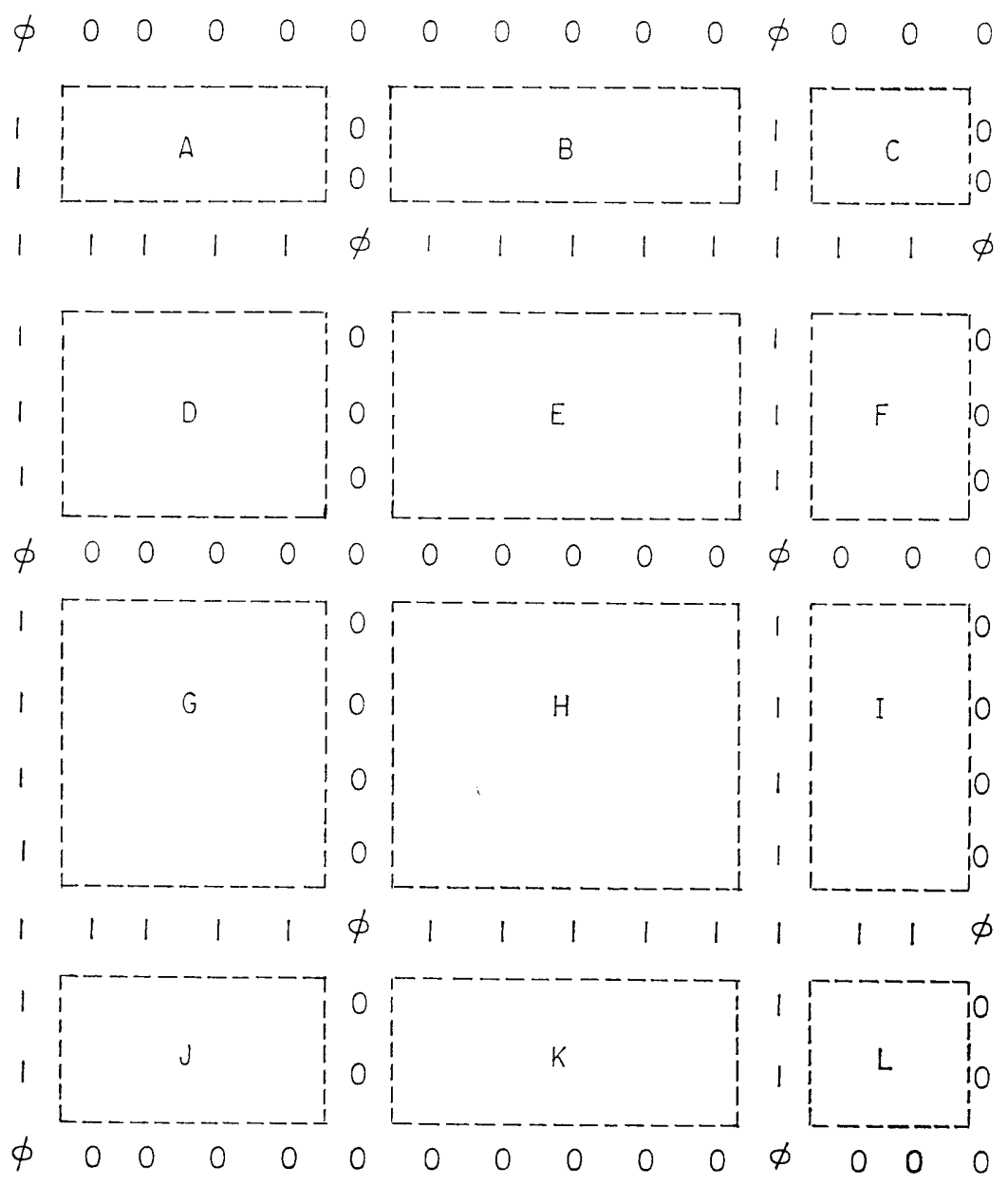

Fig. 9. $\phi$ configuration and allied subspaces.

This example shows an essential feature of any partition of cell space by use of $\phi$ states and peripherical allocations. Indeed the contents of each subspace are not uniquely determined. A characteristic feature of each partition can be shown by the following scheme of Figure 10 in which the mutual interrelations of connections among $\phi$ states, independent cell subspaces $\theta$, and peripherical values 1 and 0 are shown irrespective of size considerations.

(b) ROAD CONSTRUCTION FOR INFORMATION TRANSMISSION

We shall explain our idea again here by an example in which a state at one end of a cell space which is essentially a cell road can be carried out to the other end of the cell road. In order to convey an information at one end to the other end there is a need for introducing a change of the end configurations.

EXAMPLE 8. Let us start with the following configuration of the cell space.

The thirty one $\theta$ 's may be 1 or 0 , except that the ordinary states of $\theta_{12}$ and $\theta_{23}$ are 1 while those of $\theta_{7}$ and $\theta_{18}$ are 0 . 


$\begin{array}{ccccccccc}\phi & 0 & 0 & 0 & \phi & 0 & 0 & 0 & \phi \\ 1 & \theta & 0 & \theta & 1 & \theta & 0 & \theta & 1 \\ 1 & 1 & \phi & 1 & 1 & 1 & \phi & 1 & 1 \\ 1 & \theta & 0 & \theta & 1 & \theta & 0 & \theta & 1 \\ \phi & 0 & 0 & 0 & \phi & 0 & 0 & 0 & \phi \\ 1 & \theta & 0 & \theta & 1 & \theta & 0 & \theta & 1 \\ 1 & 1 & \phi & 1 & 1 & 1 & \phi & 1 & 1 \\ 1 & \theta & 0 & \theta & 1 & \theta & 0 & \theta & 1 \\ \phi & 0 & 0 & 0 & \phi & 0 & 0 & 0 & \phi\end{array}$

Fig. 10. $\phi$ configuration, boundary components and interior subspaces.

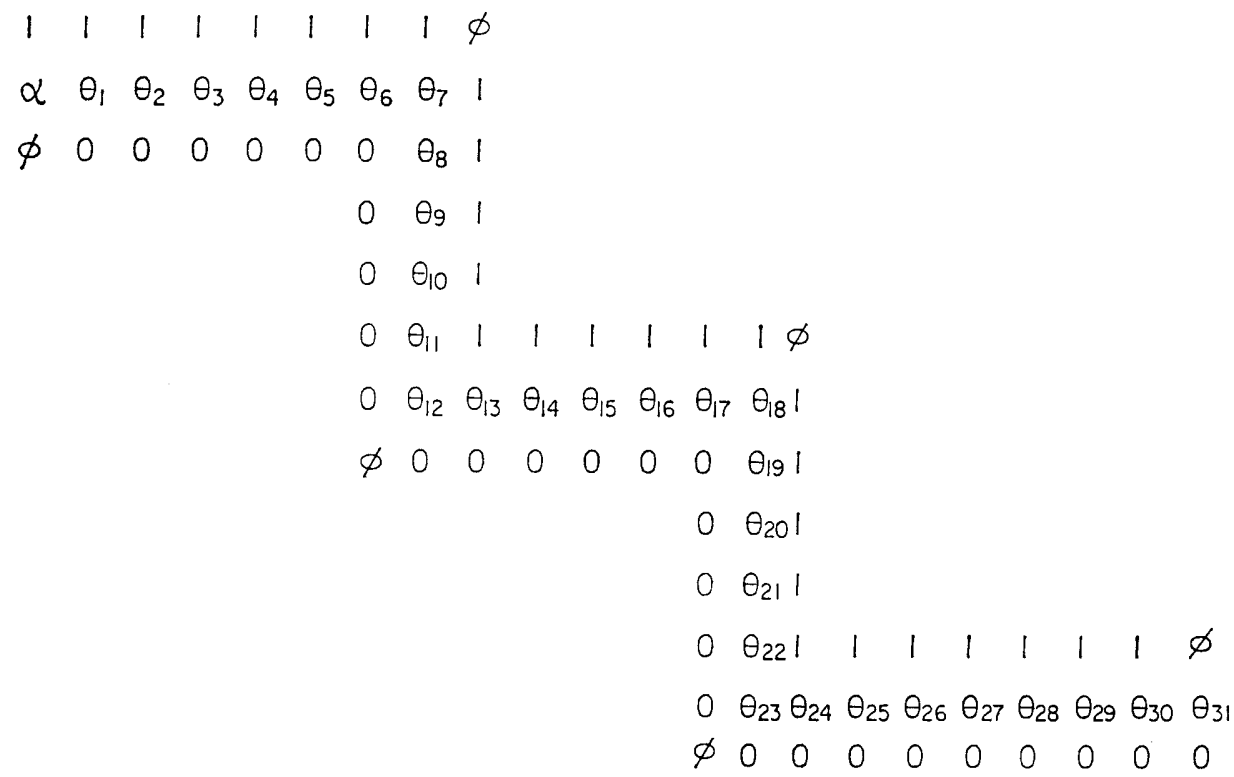

Fig. 11. Road construction by use of $\phi$ 's.

If a sequence of firing goes along the $\left\{\theta_{i}\right\}$ road in the increasing order of $i$ with a start firing as $\alpha=1$, then a sequential application of LMT's yields us $\theta_{31}=1$ since $\theta_{i}=1$ induces $\theta_{i+1}=1$ for $i=1,2,3, \cdots, 30$.

Note that there are two cells having the value 1 within the road at certain turing points.

Now let us start with another end point which corresponds to $\theta_{31}$. In stead of $\theta_{31}$ let us put $\beta$, which is now the first firing cell, and let us put $\theta_{0}$ instead of $\alpha$ at the other end. If a sequence of firing goes along the $\left\{\theta_{i}\right\}$ road in the decreasing order of $i$ with a start firing as $\beta=0$, then a sequential application of LMT's yields us $\theta_{0}=0$ since $\theta_{i}=0$ induces $\theta_{i-1}=0$ for $i=29,28,27, \cdots, 1$.

In order to convey any assigned sequence $\left(\alpha_{1} \alpha_{2} \alpha_{3} \alpha_{4} \alpha_{5} \cdots \alpha_{n} \cdots\right)$ where each $\alpha_{i}$ will take one of two values 1 and 0 , there is a need for introducing a change of the end configuration in such a way that 
(i) For $\alpha_{i}=1$, the end configuration of the road is

$$
E_{1}:\left(\begin{array}{c}
1 \\
\alpha_{i} \\
\phi
\end{array}\right)
$$

(ii) For $\alpha_{i}=0$, the end configuration of the road is

$$
E_{0}:\left(\begin{array}{c}
\phi \\
\alpha_{i} \\
0
\end{array}\right) .
$$

(iii) After passing firing, the states of $\theta_{7}, \theta_{12}, \theta_{18}$ and $\theta_{23}$ return to their tive ordinary states.

Similar consideration can be applied to the other end of the road.

(c) INFLUENCE OF DEGENERATION OF INHIBITATION STATE $\phi$

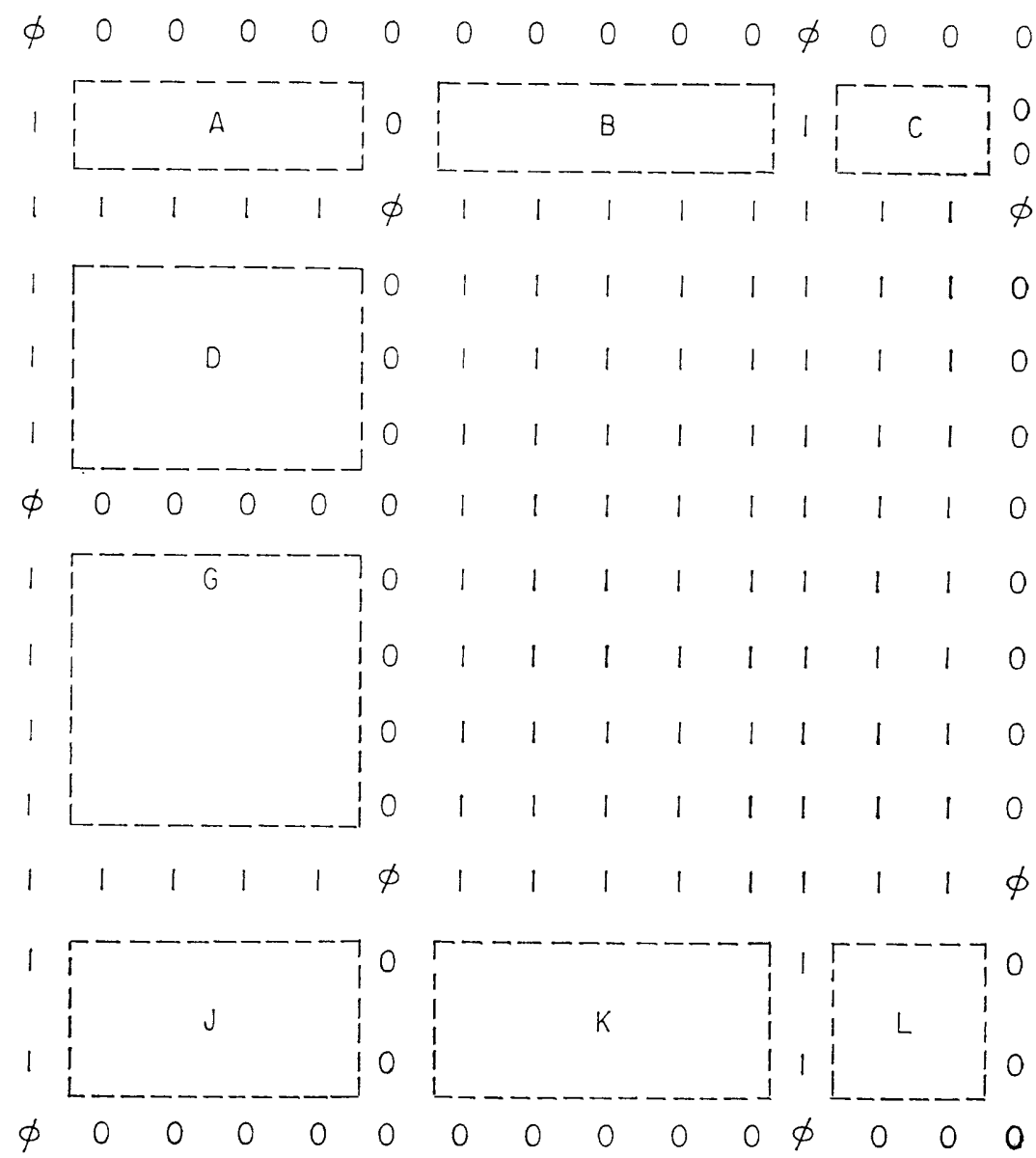

Fig. 12. Degeneration of one $\phi$ into the state 1 . 
An inhibitation state $\phi$ is in itself transcendental to any application of LMT.

However our observations given in $(a)$ and $(b)$ show that some configuration of inhibitation states may play an important role in defining a set of mutually independent cell subspaces and in introducing a communication channel which conveyes a any assigned message from the input end to the output end. In fact the existence of inhibitation states may be indispensable from the standpoint of information science approach to biomathematical model.

Now it will be interesting to observe whether and how far a modification of inhibitation functions will introduce a drastic change of configuration in cell space.

EXAMPLE 9. Let us consider again the $16 \times 15$ cell square given in Example 7 . Let us consider a change of the $(8,12)$ element $\phi$ into the state 1 . Then an applition of considerations given in Sections 2 and 3 yield us the consequence of violences of all individual subspaces $\mathrm{E}, \mathrm{F}, \mathrm{H}$ and $\mathrm{I}$ into a non-active configuration in the $8 \times 8$ cell subspace in which all elements are equal to 1.

Now let us return back to the original $16 \times 15$ cell square given in Example 7 . Let us consider a change of the $(8,12)$ element $\phi$ into the state 0 . Then the result is given by the following configuration.

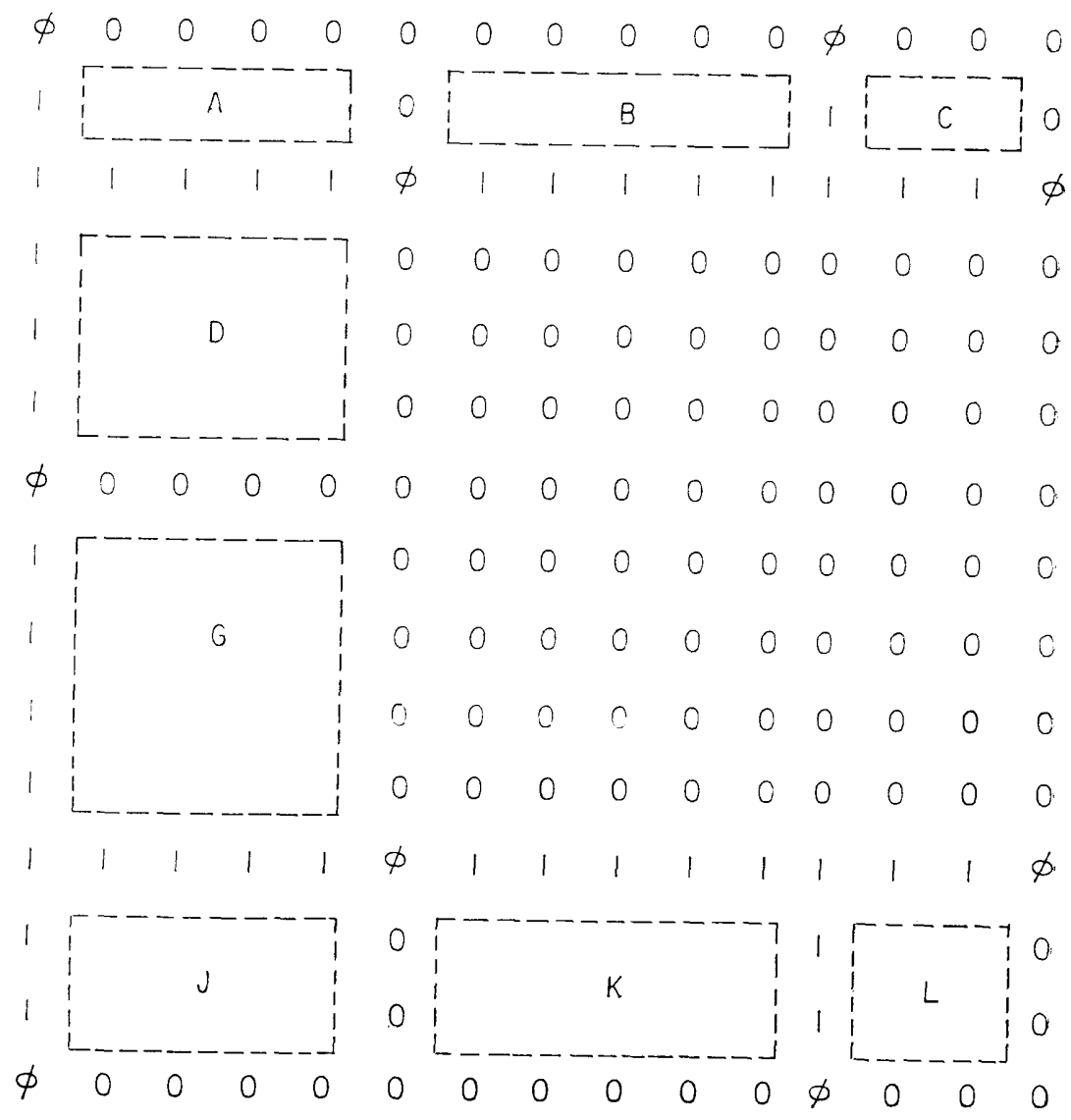

Fig. 13. Degeneration of one $\phi$ into the state 0 . 


\section{Literature}

[1] Burks, A. W. (ed): Essays on Cellular automata, Univ. of Illinois Press, Illinois, 1968.

[2] CodD, E.F.: Cellular automata, Acad. Press, New York-London, 1968.

[3] Gantmacher, F.R.: Application of theory of matrices, Translated by J.L. BRexier, Interscience Publishers, New York-London, 1959.

[4] Kitagawa, T.: Successive process of statistical contrals, (3), Mem. Fac. Sci., Kyushu Univ., Ser. A, 14 (1960), 1-33.

[5] Kitagawa, T.: A contribution to the methodology of biomathematics, Information Science Approaches to Biomathematics, 1, Research Report of the Research Institute of Fundamental Information Science, Fac. Sci., Kyushu Univ., No. 9, (1970).

[6] Minsky, M. axd Papert, S.: Perceptrons, An Introduction to Computational Geometry, M. I. T. Press, Caombridge, 1969.

[7] Moore, E. F.: Machine models of self-reproduction, Proc. Symposia Applied Math., Vol. XIV, Amer. Math. Soc., (1962), 17-34.

[8] Романовскии, Б. Й.: Дисхретные Цепи Маркова, Мосхва, 1949.

[9] Von NEUMAnn, J.: Theory of automata: construction, reproduction homogeneity, Part II of "The Theory of Self-Reproducing Automata," ed. BuRKs, A. W., Univ. of Illinois Press, Urbana, Illinois, 1966.

[10] WAng, H. A.: A variant to Turing theory of computing machines, J. Assoc. Comp. Math., 4 (1957), 63-92. 